From an Editorial perspective, this month's EMJ makes for very happy reading. The quality and breadth of original and clinically relevant research within this issue is fantastic. From large observational analyses, qualitative research embedded within multicentre clinical trials and original randomised controlled trials, to rapid original research to inform our response to COVID-19. Emergency medicine research is certainly in an excellent place advancing all the time, it is simply a pleasure to see.

\section{Too much of a good thing}

Over the past decade safe oxygen prescribing in patients with Chronic Obstructive Pulmonary Disease (COPD) attending the emergency department has vastly improved. This has been largely driven by robust trial evidence and responsive guidelines that recommend targeting oxygen saturations to $88 \%-92 \%$ in the majority of these patients. However, clinical questions remain, particularly around the use of higher targeted oxygen saturations in patients with normocapnia. In this month's Editor's Choice, an observational analysis of over 1000 patients with COPD, Echevarria and colleagues provide some compelling data. Of patients receiving oxygen, oxygen saturations $>92 \%$ were associated with increased risk of death in patients with normocapnia. The clinical implications of these data are laid out starkly in the excellent accompanying commentary, provided by Richard Beasley and colleagues, which calls for clinicians to be held to account for the excessive administration of oxygen therapy.

\section{Patient voices}

The importance of integrating qualitative work to inform randomised trial procedures and acceptability of novel clinical approaches should not be underestimated. Such elements cannot simply be captured using quantitative data. It is therefore fantastic to see three interview analyses, of qualitative studies embedded within randomised trials, LoDED (rapid discharge of low risk chest pain) and EcLiPSE (Levetiracetam or Phenytoin for status epilepticus in children), published as Open Access in the EMJ. In the LoDED qualitative analysis (disclaimer: I led this work and having had limited experience in qualitative work this was a challenge in terms of both delivery and write-up!) we explored the acceptability of very rapid discharge to both patients and clinicians (with an interesting juxtaposition) and highlight the importance of discharge communication. The EcLiPSE papers explore a key issue in emergency trials, research without prior consent, and identify barriers and enablers, together with a framework to enhance practitioner explanations, that will be key to driving forward research in the emergency setting. It's good to talk.

\section{Just when you thought sedation was boring}

I have to admit my sedation practice has become lazy and I rarely think beyond propofol and fentanyl. Familiarity breeds contempt. It is therefore great to see two original articles that challenge my engrained clinical practice. This month's Reader's Choice is a multicentre feasibility study of propofol target-controlled infusion for sedation in the ED from colleagues in the West of Scotland. Although primarily focused on establishing the feasibility of a large randomised trial, this work also explores potentially important patient reported outcomes for sedation research. We look forward to seeing whether the authors can incorporate patient reported outcomes into a future definitive trial. In our second sedation paper this month, Rasooli et al compare Ketamine-Propofol and Sodium Thiopental-Fentanyl in a randomised controlled trial. With impressive blinding strategies, including opaque coverage of syringes, they found in 96 patients that both strategies were equally efficacious, but with higher patient satisfaction in the ketaminepropofol group.

\section{COVID-19}

In 2020, submis-

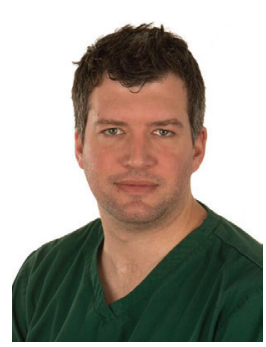
sions to the EMJ

(total 1560) nearly doubled from 2019 (total 863). This is testament to the drive and enthusiasm for emergency physicians to create an evidence base for the response to COVID-19. Unfortunately, not all submissions can progress to publication and acceptance rates to the journal have remained the same. This month, we publish four manuscripts which focus on various clinical aspects of COVID-19 from airway and ventilator management to novel service adaptions including telemedicine and remote patient monitoring.

\section{The challenge of the Breathless patient}

Diagnosis of the undifferentiated breathless patient in ED is perhaps more challenging than ever. Proponents of point of care ultrasound may often reach for the probe to guide clinical management in these patients. However, in a systematic review and metaanalysis published this month by Squizzato and colleagues, which explores the diagnostic accuracy of inferior vena cava ultrasound for heart failure, the authors question its accuracy as a stand-alone test (mean specificity of $81.8 \%$, in 7 studies including 591 patients in total). While using ultrasound in isolation may be problematic, the authors suggest it retains a role when integrated into broader diagnostic algorithms.

\section{ORCID iD}

Edward Carlton http://orcid.org/0000-0002-2064-4618 